\title{
Extraction of Bio Polymers from Crustacean Shells and its Application in Refinery Wastewater Treatment
}

\author{
Khadija Salim ABDULLAH AL BALUSHI, Geetha DEVI*, \\ Amira Sulaiman RASHID KHAMIS AL GHARIBI, \\ Mohammed Adeeb Salim ADEEB, Alaa Saif Mohammed AL HUDAIFI and \\ Siham Salim KHALFAN AL SHABIBI
}

Department of Mechanical \& Industrial Engineering, College of Engineering, National University of
Science and Technology, Sultanate of Oman, Oman

('Corresponding author’s e-mail: geethadevi@nu.edu.om)

Received: 1 August 2020, Revised: 25 November 2020, Accepted: 19 December 2020

\begin{abstract}
The fisheries sector is one of the most ancient and important sectors in the world and plays a significant role in providing the nutrition and socio-economic development of the country. The fish processing industry produces huge quantities of wastewater, encompassing significant amounts of contaminants in the form of soluble, colloidal, and particulate matters. The disposal of shellfish waste is a serious issue, and the effluents discharged from seafood-processing plants contain high amounts of Chemical Oxygen Demand (COD), Biochemical Oxygen Demand (BOD), dissolved solids, suspended solids, and turbidity. The existing waste management system lacks a cost-effective and environmentally friendly method. The current research focus on the extraction of a biopolymer chitosan from crab shells by ecofriendly methods and its application in refinery wastewater treatment. The chemical structure and crystallinity of the extracted chitosan was confirmed by Fourier Transform Infrared Spectroscopy (FTIR) and X-Ray Diffraction (XRD) analyses. Surface morphology and elemental composition were determined using Scanning Electron Microscopy (SEM), and Energy-Dispersive X-Ray Analysis (EDX). Thermal properties were detected using Thermo Gravimetric Analysis (TGA). The extracted chitosan was successfully employed in the batch treatment of refinery effluent by varying the experimental parameters (refinery effluent solution $\mathrm{pH}$, contact time, dosage of chitosan, and stirring speed) and the optimizations of the processing conditions were established.
\end{abstract}

Keywords: Biochemical oxygen demand, Chitosan, Crab shell, Refinery effluent, Thermo gravimetric analysis

\section{Introduction}

Biopolymers are natural polymers derived from their natural sources, which assumed to be renewable and replenishable. Nearly $45 \%$ of processed seafood consists of crustacean shells (crab shells, prawn shells etc.), of which $50-70 \%$ of the weight of the raw material contains chitin, a protein. Chitin is the $2^{\text {nd }}$ most abundant natural polysaccharide found in nature after cellulose [1,2] and exists in the shell wall of marine invertebrates, insects, certain fungi and algae [3,4]. The main sources of chitin are principally crabs and shrimps [5], from which the biopolymer chitosan is derived [6]. The attractive features of chitin and chitosan are their excellent biodegradability, biocompatibility, non-toxicity, antimicrobial and antioxidant activities, and wound healing capacities, which make them suitable in biomedical and pharmaceutical fields, drug delivery, cancer diagnosis, tissue engineering, wound dressing (artificial skin), and water treatment applications [7,8]. 
The processing stages of crustacean shells mainly include alkaline extraction in the deproteinization step, followed by acid treatment to dissolve calcium carbonate, which is present in high concentrations in shells $[1,9]$. Chitosan is a poly cationic polymer obtained by the deacetylation of chitin. The principal sources of production of chitin are crabs and shrimps. Chitosan is mainly applied in water treatment and purification because of its adsorption and chemical properties [11]. The consumption of crabs in Oman is on the higher side, and a considerable amount of crab shells are discarded into the environment as waste, which causes serious environmental problems. Hence, it is required to process the waste crab shells and convert them into value added products.

The coagulation, flocculation, cost-effective, and environmentally-friendly properties of chitosan have gained considerable attention in the area of wastewater treatment applications [12-18]. Themajority of the conventional approaches for the extraction of chitin and chitosan from crustaceans use chemical processes utilizing strong acids and bases for demineralization and deproteination steps. These processing steps generate huge environmental pressure for the treatment of wastewater, and also utilize huge quantities of fresh water for cleaning and washing steps. It has been reported that chemical purification of chitin is extremely hazardous, energy demanding, and damaging to the environment. In order to avoid these issues, the current research focuses on an environmentally-friendly and cost-effective method to extract chitin and chitosan from crab shell, to be used instead of the traditional extraction method employing harsh chemicals and acids.

Thus, the present study attempts to examine the green extraction of biopolymers from crab shells under mild experimental conditions, in order to reduce chemical usage, lower energy consumption, and protect the environment. The extracted chitosan was employed in the treatment of refinery wastewater in a safe and environmentally-friendly way.

\section{Materials and methods}

\section{Raw materials}

Crab shells were collected from a local fish processing plant located in Muscat. The shells were washed several times with fresh water, followed by drying in a hot air oven at $90{ }^{\circ} \mathrm{C}$ for $30 \mathrm{~min}$. The dried samples were ground to fine powder using a ball bill, and the average particle size was measured by sieve analysis. Particles of size less than $75 \mu \mathrm{m}$ were used for the extraction experiment. Citric acid, nitric acid, sodium hydroxide pellets, and acetone were purchased from Chemistry for Life Company, Oman. The refinery effluent samples were collected from the Occidental Company, Oman. The chemicals used for the experiment were of analytical grade and used without further purification. All experiments were repeated 3 times, and the averages of 3 values were considered as the final values.

The characterization techniques employed were Scanning Electron Microscopy (SEM JEOL JSM7600F), X-Ray diffractometry (Rigaku, Mini Flex 600), Fourier Transform Infrared Spectroscopy (FTIRPerkin Elmer Frontier), Thermo Gravimetric Analysis (TGA), and Energy Dispersive X-Ray Analysis (EDX). All samples used for EDX analysis were coated with gold to prevent the accumulation of static electric fields during imaging. Thermo gravimetric analysis was performed to determine the mass of sample over a range of 30 to $900^{\circ} \mathrm{C}$.

\section{Extraction of chitosan using organic acid}

The extraction process was carried out by mixing powdered crab shell with $1.0 \mathrm{~N}$ citric acid. The crab shell powder and citric acid were mixed at a ratio of 1:1.5and theextraction process was carried out at room temperature for $1 \mathrm{~h}$. The resulting mixture was washed with distilled water, followed by centrifugation at 5,000 rpm for $5 \mathrm{~min}$, and was then dried at $100{ }^{\circ} \mathrm{C}$ in a hot air oven. The dried samples were deproteinized by dissolving in $0.1 \mathrm{~N}$ sodium hydroxide solutions at $80{ }^{\circ} \mathrm{C}$ for $6 \mathrm{~h}$, followed by sequential washing with distilled water until $\mathrm{pH}$ reached 7.0, and was then filtered and dried to $60^{\circ} \mathrm{C}$. The color of the powder was removed using acetone under continuous stirring for $24 \mathrm{~h}$ at a speed of $100 \mathrm{rpm}$. The sample after color removal was deacetylated to get chitosan. The acetyl groups were removed by dissolving $1.0 \mathrm{~g}$ of powdered sample in $10 \%$ sodium hydroxide solution and stirring for $6 \mathrm{~h}$ at $85{ }^{\circ} \mathrm{C}$, 
followed by washing with distilled water and drying in an oven at $60{ }^{\circ} \mathrm{C}$. The resulting samples were preserved for characterization and analysis using SEM, FTIR, XRD, TGA, and EDX, respectively.

\section{Treatment of refinery effluent using chitosan}

The extracted chitosan was employed in the batch treatment of refinery wastewater by varying the effluent solution $\mathrm{pH}$, mixing time, agitation speed, and dosage of chitosan.

\section{Effect of variation of refinery effluent solution $\mathrm{pH}$}

The influence of change in effluent solution $\mathrm{pH}$ on the removal of contaminants was studied by adjusting the $\mathrm{pH}$ of the refinery effluent from 2.0 to 7.0. The experiment was accomplished by mixing 0.2 $\mathrm{g}$ of chitosan powder with $150 \mathrm{~mL}$ of refinery effluent and stirring for $45 \mathrm{~min}$ with a stirring speed of 25 rpm. The resulting mixture was tested for TDS, TSS, COD and BOD, and turbidity.

\section{Effect of variation of mixing time}

Mixing time plays a significant role in pollutant removal. The effect of variation of mixing time in the reduction of parameters was studied by varying the contact time from 15 to $90 \mathrm{~min}$. The $\mathrm{pH}$ of the effluent solution was maintained at 7.0 and a stirring speed of $25 \mathrm{rpm}$.

\section{Effect of variation of dosage of chitosan}

The effect of variation of dosage of chitosan on pollution removal was studied by changing the amount of chitosan from 0.1 to $0.5 \mathrm{~g}$, with an optimized $\mathrm{pH}$ of the effluent and a mixing time.

\section{Effect of variation of agitation speed}

Effect of agitation speed of chitosan on contaminant removal efficiency was investigated by changing the stirring speed from 25 to $125 \mathrm{rpm}$, keeping all other parameters constant.

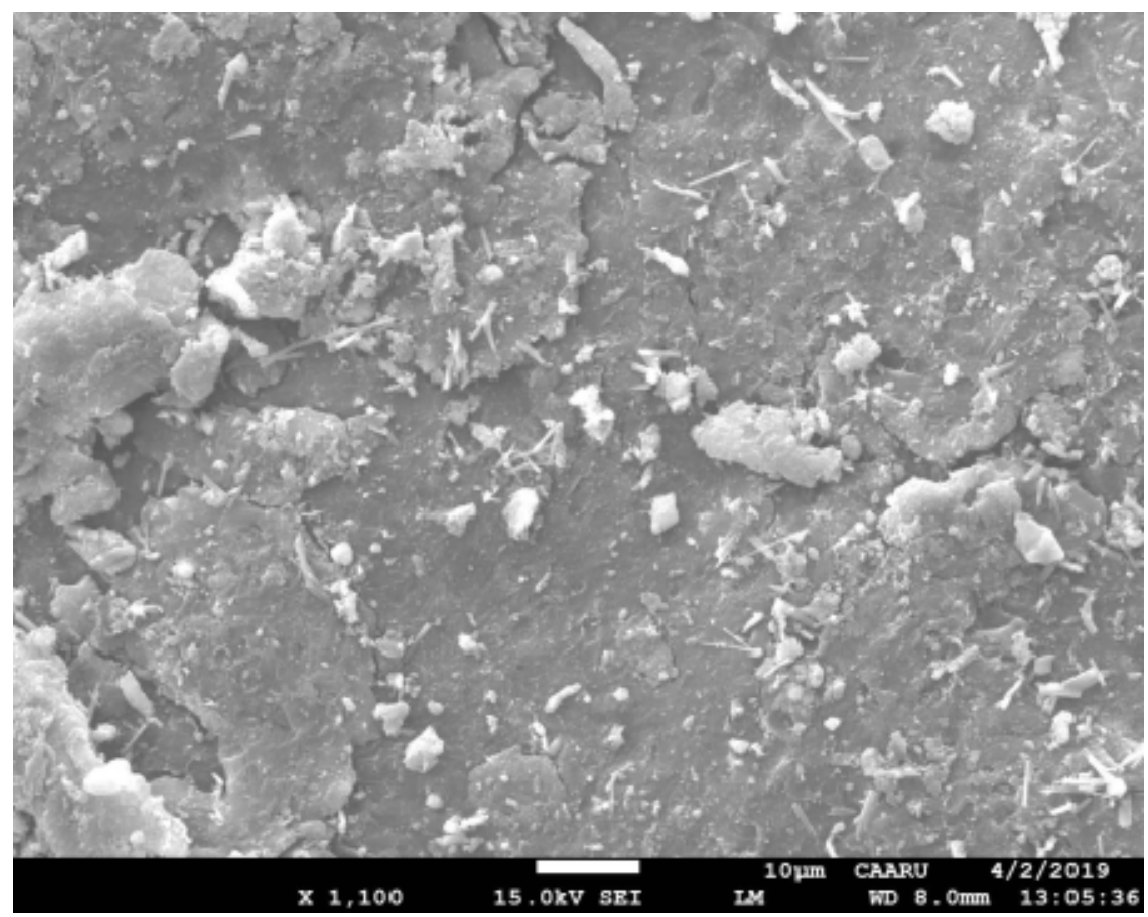

Figure 1 SEM image of chitosan at a magnification of 1,100 X. 


\section{Results and discussion}

\section{Surface characterization of chitosan}

Scanning Electron Microscopy (SEM) analysis

The surface characterization of the extracted chitosan powder was performed using scanning electron microscopy, with the image is displayed in Figure 1. The SEM image indicates that the chitosan powder was distributed evenly without aggregation, which designates the successful extraction of chitosan. The morphological characterization of chitosan powder, illustrated in Figure 1, confirmed that the particles were scattered with non-homogeneous distribution, even though the size and shape of the particles were uneven. The samples exhibited rough and thick surface structure under scanning electron microscopic examination at a magnification of $1,100 \mathrm{X}$ and an excitation voltage of $15.0 \mathrm{kV}$, as depicted in Figure 1.

\section{SEM-EDX analysis}

The elemental composition of the chitosan sample obtained from SEM-EDX analysis is shown in Figure 2. The elemental analysis indicated $42.8 \%$ Carbon, $40.8 \%$ Oxygen, $8.6 \%$ Nitrogen, $4.2 \%$ Sodium, $2.2 \%$ Calcium, $0.9 \%$ Magnesium, and $0.5 \%$ Chlorine, respectively.

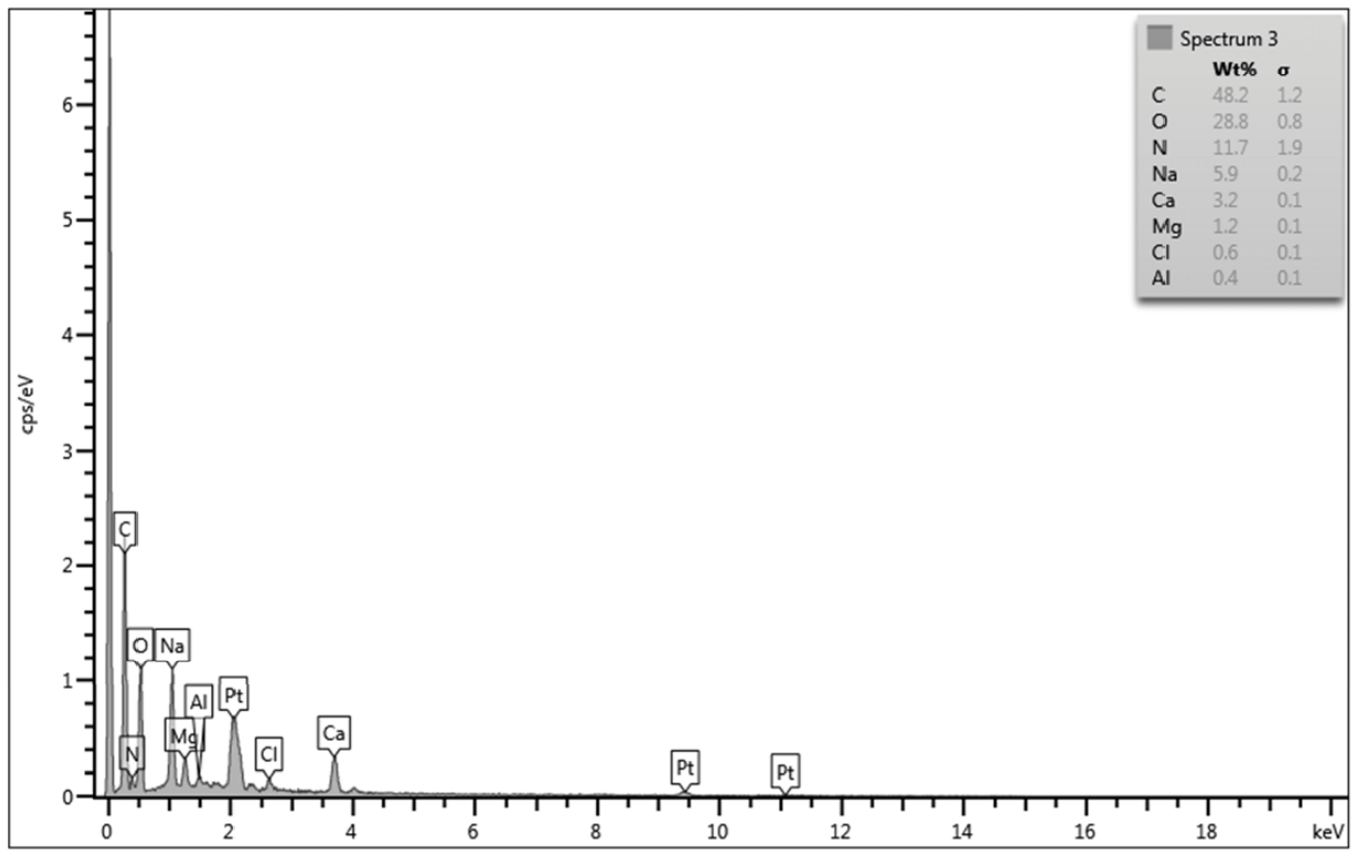

Figure 2 SEM-EDX elemental composition analysis of chitosan.

\section{X-Ray diffraction analysis}

The crystalline structures of the extracted chitosan powder are presented in Figure 3. The crystalline reflections were analyzed at a scan rate of $1 \% / \mathrm{min}$ with scan angle ranges from 2 to $80^{\circ}$. The XRD pattern illustrates the crystalline nature of chitosan powder, and the diffraction peaks are observed at $2 \theta$ between $10-15^{\circ}$ and $25-30^{\circ}$. The result was in agreement with the previous studies [19,20]. The extra peaks represent the nature of solvents used in the demineralization and deproteinization stages. The reflection peak indexed at $25^{\circ}$ confirmed the presence of chitosan. 


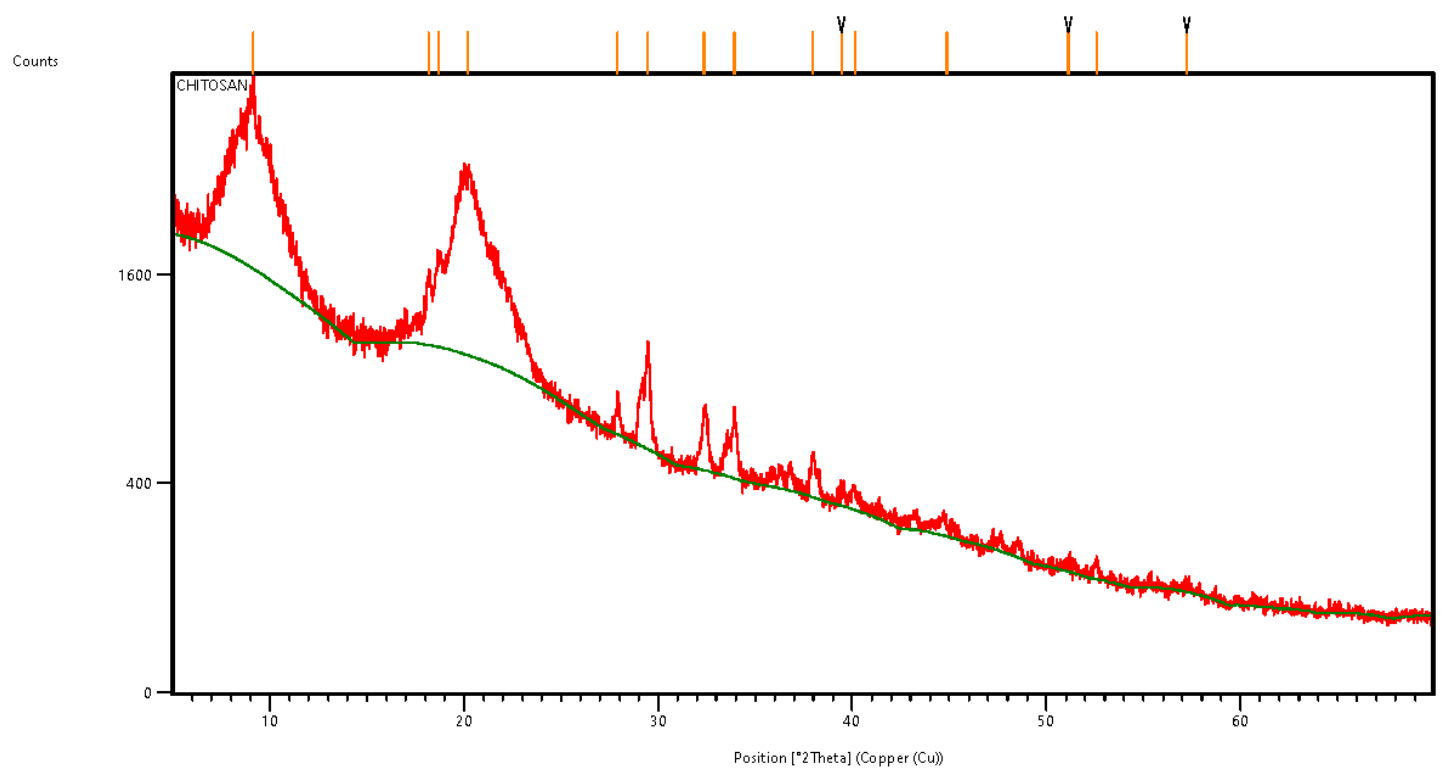

Figure 3 X-Ray Diffraction pattern of chitosan powder.

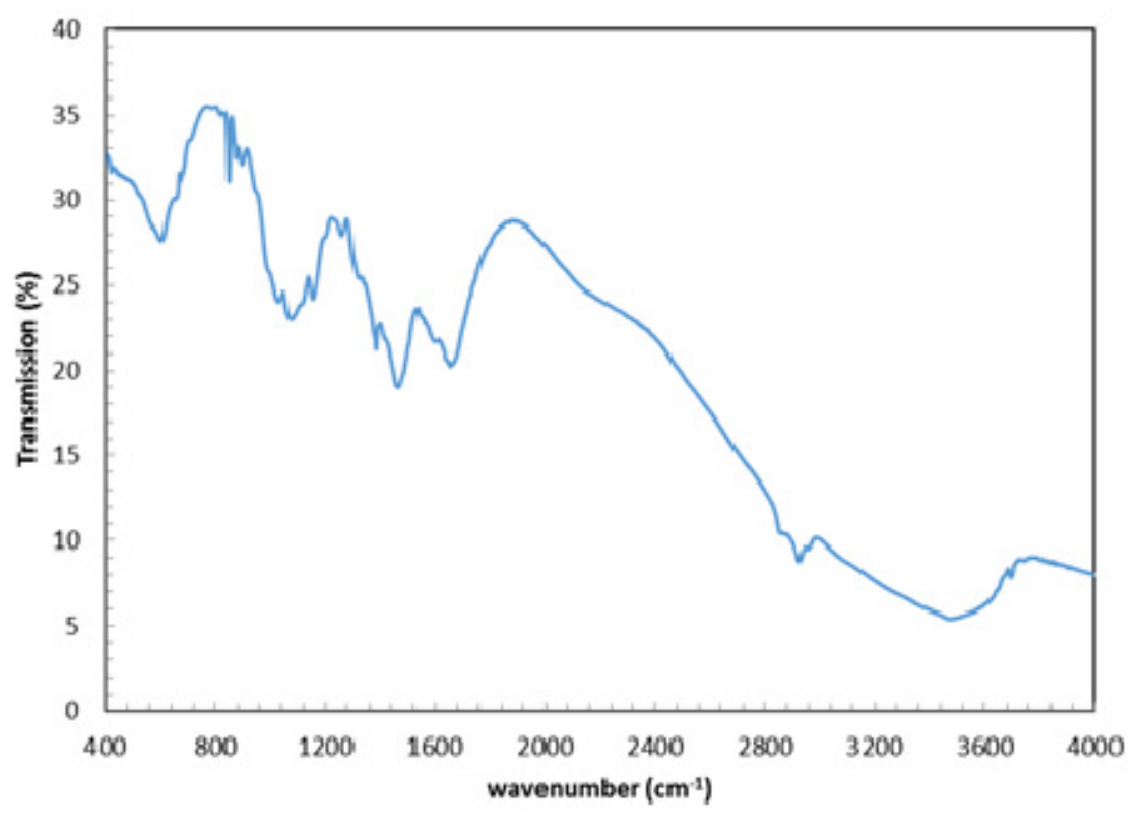

Figure 4 FTIR Spectra of chitosan powder.

\section{Fourier Transform Infrared Spectroscopy (FTIR):}

The FTIR spectroscopic analysis of chitosan powder was obtained at a frequency range between 400 to $4,000 \mathrm{~cm}^{-1}$ and at a resolution of $4 \mathrm{~cm}^{-1}$. The presence of functional groups and bond stretching present in the chitosan powder are indicated in Figure 4. The characteristic peaks of $\mathrm{O}-\mathrm{H}, \mathrm{C}-\mathrm{H}, \mathrm{C}=\mathrm{O}$, $\mathrm{CH}=\mathrm{H}, \mathrm{CH}_{2} \mathrm{OH}$, and $\mathrm{N}-\mathrm{H}_{2}$ stretching are seen at wave numbers corresponding to $3,400,2,930,1,659,1,468$, 
1,380 and 1,680 $\mathrm{cm}^{-1}$, respectively. The $\mathrm{N}-\mathrm{H}$ and the $\mathrm{O}-\mathrm{H}$ stretching bands of chitosan were observed between 800 to $1,600 \mathrm{~cm}^{-1}$. The FTIR spectra shown in Figure 4 match with the previous studies [21].

\section{Thermo Gravimetric Analysis (TGA)}

The thermal degradation temperature of extracted chitosan was examined using TGA with 2 major degradation steps, as shown in Figure 5. From Figure 5, it was observed that the $1^{\text {st }}$ stage of degradation of chitosan occurred between 30 and $90{ }^{\circ} \mathrm{C}$, with nearly $4 \%$ weight loss. The $2^{\text {nd }}$ stage was between 190 and $270{ }^{\circ} \mathrm{C}$, with $60 \%$ weight loss. This degradation process may have been due to the loss of moisture or water molecules from chitosan. The TGA curves shift toward higher temperatures along with growth in heating rate.

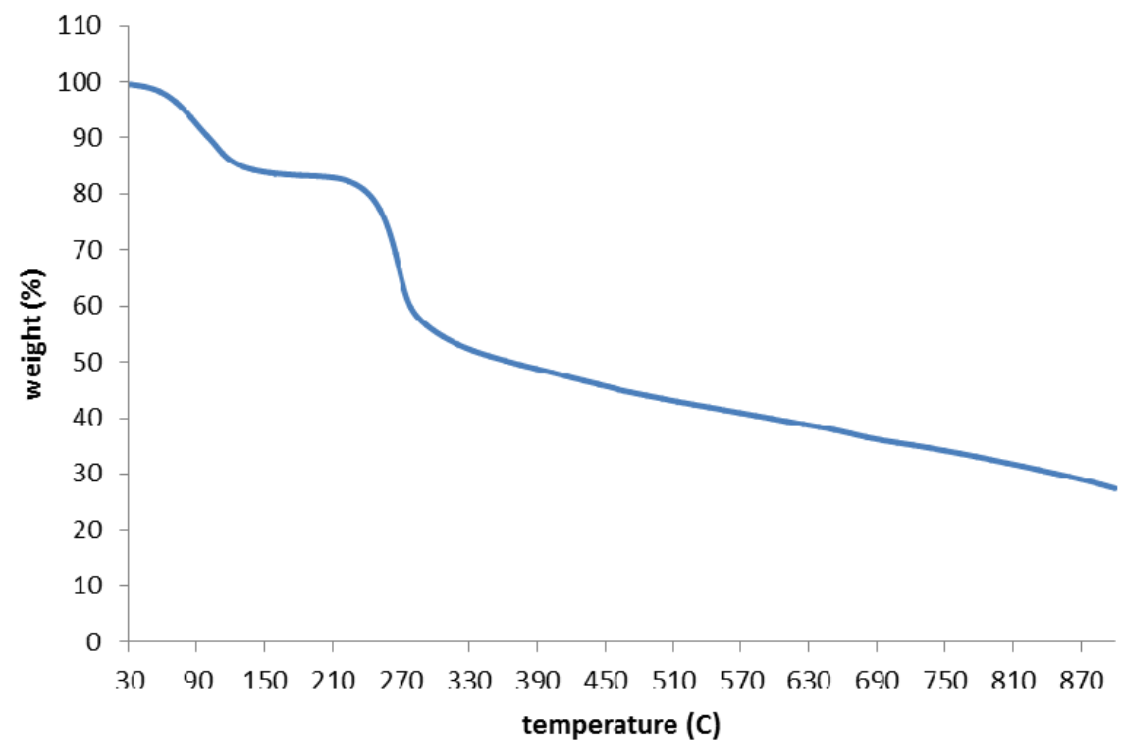

Figure 5 TGA Curves of thermal degradation of extracted chitosan powder.

\section{Refinery wastewater treatment}

\section{Effect of refinery effluent solution $\mathrm{pH}$}

After the batch adsorption process, the resulting mixture was tested for TDS, TSS, COD, BOD, and Turbidity. The maximum reduction in parameters was obtained at an optimum solution $\mathrm{pH}$ corresponding to 6.0. The highest reductions in parameters were obtained due to the coagulation and flocculation properties of chitosan. The surface charge of chitosan was highest and, hence, the adsorption property also increased, resulting in the destabilization of pollutants in the wastewater. This could be achieved due to the adsorption of excess polymer on the surfaces. The charge transfer caused an electrostatic repulsion in the suspended solids, leading to reduction in the COD. Optimum $\mathrm{pH}$ was found to be 6.0 , with a percentage reduction of COD as $78 \%$. Figure 6 represents the effect of variation of refinery effluent solutionpH with parameter reductions. 


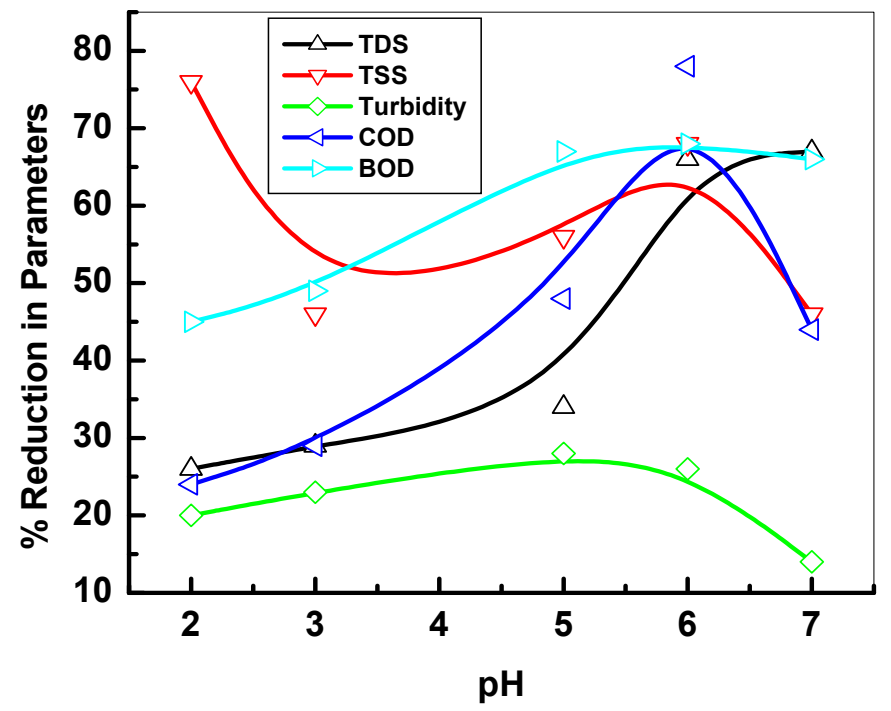

Figure 6 Effect of variation of refinery effluent solution $\mathrm{pH}$ with parameter reductions.

\section{Effect of mixing time}

As mixing time increased, the development of flocs increased and the flocculent started dispersing throughout the medium over a certain period of time. The extended mixing time enhanced the breakage of flocs into smaller ones, thereby retarding flocculation rate. The ideal contact time for the maximum reduction of COD was observed at $90 \mathrm{~min}$, with a percentage reduction corresponding to $53 \%$. Figure 7 illustrates the influence of contact time on parameter reductions. The percentage reduction in TDS was in an increasing order up to a contact time of $60 \mathrm{~min}$, beyond which the percentage reduction diminishes.

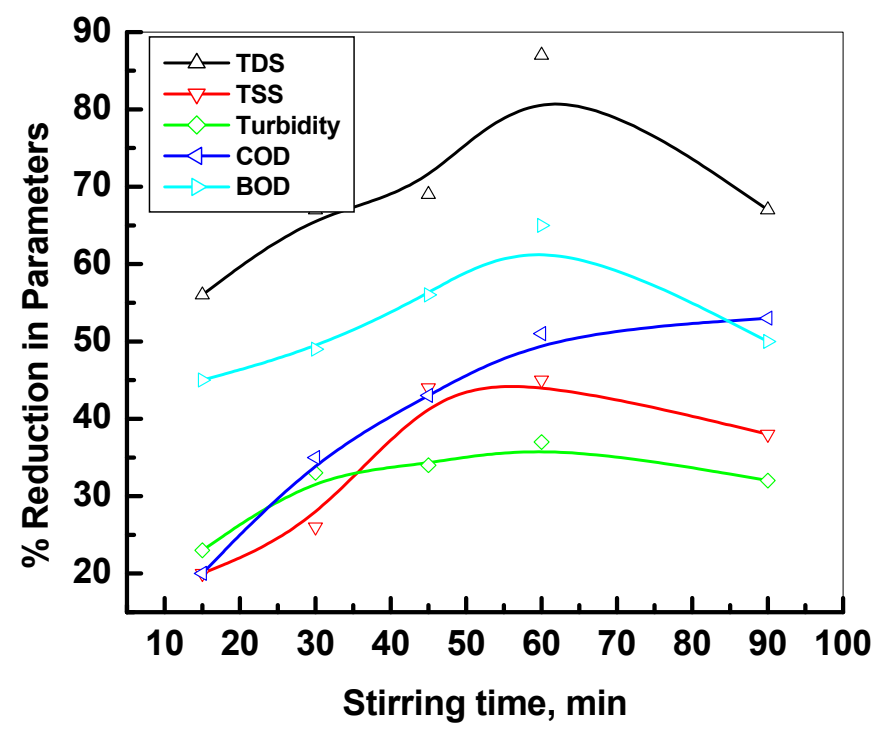

Figure 7 Effect of variation of mixing time with parameter reductions. 


\section{Effect of dosage of chitosan}

The slow addition of chitosan powder into the effluent solution ensured an increase in the \% reduction of TDS. The reduction in TDS was found to increase steadily due to the availability of more adsorption sites on the surface of chitosan, which enhanced the deposition tendency of pollutants. This may have been due to the strong molecular interaction between the amine groups present in chitosan and the pollutants present in the effluent. The \% reduction in TSS showed a fluctuating behavior due to the formation of bigger flocs in the mixture upon changing the dosage of chitosan. As dosage increased, the turbidity reduction tended to decrease. The positive charge of chitosan with free amine groups electrostatically interacted with the negative charge of pollutants present in the wastewater. This lead to instability and to turbidity reduction. The \% reduction in COD steadily increased up to $0.4 \mathrm{~g}$ of chitosan dosage and then decreased. This was due to the electrostatic repulsion between particles. The effects of variation of dosage of chitosan with pollutant removal efficiency are shown in Figure 8.

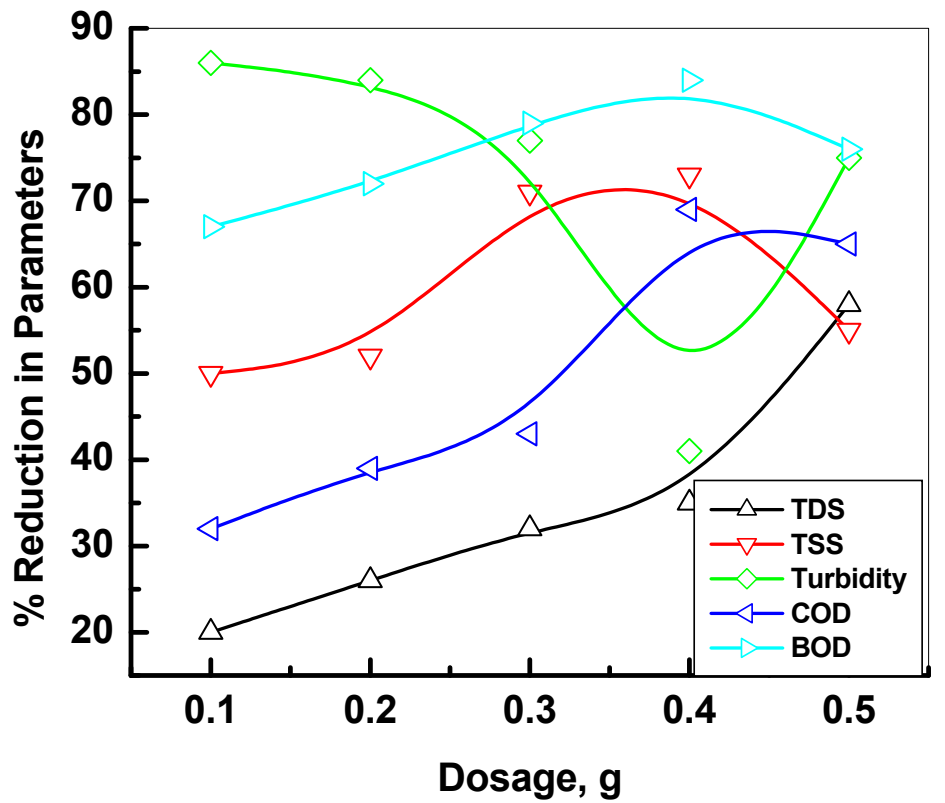

Figure 8 Effect of variation of dosage of chitosan with parameter reductions.

\section{Effect of agitation speed}

As the speed of agitation increased, the particle size tended to decrease, thereby creating an enriched surface area which allowed improved adsorption efficiency. The \% reduction in TDS was found to increase with increase in stirring speed. The optimum reduction in TDS was obtained at a stirring speed of $100 \mathrm{rpm}$. The percentage reduction in COD increased with increase in stirring speed with the range of values studied, whereas the turbidity decreased with increase in stirring speed, as indicated in Figure 9. The percentage reduction in TSS increased with increasing speed up to $75 \mathrm{rpm}$, followed by a sudden decrease in removal efficiency. 


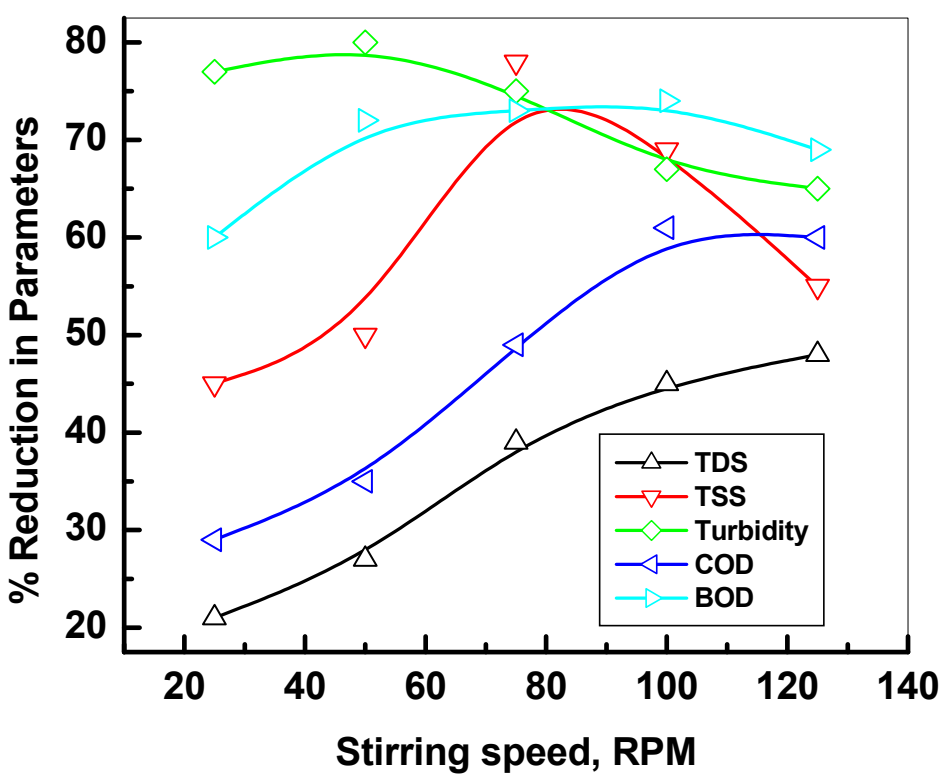

Figure 9 Effect of variation of stirring speed with parameter reductions.

\section{Conclusions}

In this research, a sustainable, ecofriendly, and green technique was employed for the extraction of chitosan from crab shell at elevated temperature. The surface morphology, structural characterization, composition analysis, and functional groups of the extracted chitosan were characterized using SEM, EDS, FTIR, XRD, and TGA. The X-Ray Diffraction analysis revealed a peak at 25 - 30 degrees, indicating the successful extraction of chitosan. The Fourier Transform Infrared (FTIR) analysis demonstrated the absence of acetyl groups and the breakage of glucosidic bonds. The extracted chitosan was successfully employed in the batch treatment of refinery wastewater by varying the processing conditions, and the optimum percentage reductions in parameters were determined. This research will serve as an incentive for fish processing industries, as it involves an environmentally-friendly approach for the extraction of chitosan and also minimizes marine pollution. Extraction of chitosan from waste crab shells would help to solve the environmental issues associated with fish processing industries, improve water quality, create green environments, and help reduce health hazards. It would also help to increase the country's per capita income, by exporting the extracted chitosan to suitable customers. In addition, this research will transform solid waste into a value-added and environmentally-friendly product. This environmentally-friendly approach for the green extraction of chitosan will be a better option for marine waste management without using harsh chemicals. Currently, the research team is exploring the possibility of extending the experimental work in column studies using textile mill effluent and dairy wastewater treatment to optimize the processing conditions and to compare the efficiency. This community engagement project will address 3 basic issues of human society- water, energy, and pollution- with a strong interdisciplinary lens ranging from engineering, ecology, and social work. 


\section{Acknowledgements}

The authors express their sincere thanks to The Research Council (TRC) Oman for awarding financial support for the research under the Graduate Research Grant program (Ref. No. BFP/GRG/EBR/19/008CoE). Also, special thanks to the Dean and Deputy Deans of the College of Engineering, National University of Science \& Technology, Oman, in providing the infrastructure facilities to carry out the experiments at the chemical engineering laboratory.

\section{References}

[1] RM Younus. Chitin and Chitosan preparation from marine sources: Structure, properties and applications. Mar. Drugs 2015; 13, 1133-74.

[2] E Bulut, I Sargin, O Arslan, M Odabasi, B Akyuz and M Kaya. In situ chitin isolation from body parts of a centipede and lysozyme adsorption studies. Mater. Sci. Eng. C 2017; 70, 552-63.

[3] CC Chen, YR Wang, YN Yang, MZ Pan, T Ye and DG Li. High strength gelatin-based nano composites reinforced by surface-deacetylated chitin nano fiber networks. Carbohydr. Polym. 2018; 195, 387-92.

[4] Y Huang, M Yao, X Zheng, X Liang, X Su, Y Zhang, A Lu and L Zhang. Effects of chitin whiskers on physical properties and osteoblast culture of alginate based nano composite hydrogels. Biomacromolecules 2015; 16, 3499-507.

[5] RA Kumar, A Sivashanmugam, S Deepthi, S Iseki, KP Chennazhi, SV Nair and R Jayakumar. Injectable Chitin-poly ( $\mathcal{E}$-Caprolactone)/nano hydroxyapatite composite microgels prepared by simple regeneration technique for bone tissue engineering. ACS Appl. Mater. Interfaces 2015; 7, 9399-409.

[6] S Hajji, O Ghorbelbellaaj, I Younes, K Jellouli and M Nasri. Chitin extraction from crab shells by Bacillus bacteria: Biological activities of fermented crab supernatants. Int. J. Biol. Macromol. 2015; 79, 167-73.

[7] P Zhu, Z Gu, S Hong and H Lian. One-pot production of chitin with high purity from lobster shells using choline chloride-malonic acid deep eutectic solvent. Carbohydr. Polym. 2017; 177, 217-23.

[8] R Zhu, C Zhang, Y Liu, ZQ Yuan, WL Chen, SD Yang, JZ Li, WJ Zhu, XF Zhou, BG You and XN Zhang. CD147 monoclonal antibody mediated by chitosan nanoparticles loaded with $\alpha$-hederin enhances antineoplastic activity and cellular uptake in liver cancer cells. Sci. Rep. 2016; 5, 1-11.

[9] H Li and LH Greene. Sequence and structural analysis of the chitinase insertion domain reveals two conserved motifs involved in chitin-binding. PLoS One 2010; 1, 1-11.

[10] N Yaghobi and H Mirzadeh. Enhancement of chitin's degree of deacetylation by multistage alkali treatments. Iran. Polym. J. 2004; 13, 131-6.

[11] OBS Hasan, S Satheesh and MA Ba-akdah. Extraction of chitin from the shell wastes of two shrimp species Fenneropenaeus semisulcatus and Fenneropenaeus indicus using microorganisms. J. Aqua. Food Prod. Tech. 2016; 26, 390-405.

[12] J Bratby. Coagulation and flocculation in water and wastewater treatment. $2^{\text {nd }}$ eds. IWA Publishing, 2007, p. 1-450.

[13] VR Giri-Dev, R Neelakandan, S Sudha, OL Shamugasundram and RN Nadaraj. Chitosan: A polymer with wider applications. Text. Magaz. 2005; 46, 83-6.

[14] KVH Prashanth and RN Tharanathan. Chitin/Chitosan: Modifications and their unlimited application- an overview. Trends Food Sci. Technol. 2007; 18, 117-31.

[15] N Kashyap, N Kumar and MNVR Kumar. Hydrogels for pharmaceutical and biomedical applications. Crit. Rev. Ther.Drug. Carrier Sys. 2005; 22, 104-50.

[16] B Krajewska. Membrane-based processes performed with use of Chitin/Chitosan materials. Sep. Purif. Technol. 2005; 41, 305-12.

[17] F Renault, B Sancey, PM Badot and G Crini. Chitosan for coagulation/flocculation processes-an eco-friendly approach. Eur. Polym. J. 2009; 45, 1337-48. 
[18] C Dong, W Chen and C Liu. Flocculation of algal cells by amphoteric chitosan-based flocculant. Bioresour. Technol. 2014; 170, 239-47.

[19] MT Yen, JH Yang and JL Mau. Physico chemical characterization of chitin and chitosan from crab shells. Carbohydr. Polym. 2009; 75, 15-21.

[20] RAA Muzzarelli, P Morganti and G Morganti. Chitin nanofibrils/Chitosan glycolate composites as wound medicaments. Carbohydr. Polym. 2007; 70, 274-84.

[21] M Rinaudo. Chitin and Chitosan: Properties and applications. Prog. Polym. Sci. 2006; 31, 603-63. 